\title{
Minireview
}

nephron

Clinical

Practice

Published online: December 19, 2014

DOI: $10.1159 / 000369817$

\section{Uremic Toxins and Their Effects on Multiple Organ Systems}

\author{
Barbara Lisowska-Myjak \\ Department of Biochemistry and Clinical Chemistry, Medical University of Warsaw, Warsaw, Poland
}

\section{Key Words}

Acute kidney injury - Chronic kidney disease .

Uremic compounds $\cdot$ Renal dysfunction

\begin{abstract}
Nearly all body organs and systems are affected by the toxicity of uremic compounds retained in the course of renal dysfunction. Knowledge about the origin, chemical structure and composition of the retained endogenous substances responsible for these symptoms is far from complete. Organic retention solutes present a great variety of properties which makes their accurate classification extremely difficult. Their potential toxicity remains to be elucidated with meticulous observation of clearly formulated rules guiding the process. Toxicity assessment is a complex process because not just one but several retained compounds may be simultaneously involved in the same biological and metabolic processes. The search for new uremic compounds and combining them into panels of substances involved in the same pathophysiological processes seems to offer a novel approach to identifying and explaining any so far unexplored specific effects of endogenous compounds on the body organs and systems.

(c) 2014 S. Karger AG, Basel
\end{abstract}

(c) 2014 S. Karger AG, Basel

$1660-2110 / 14 / 1284-0303 \$ 39.50 / 0$

\section{Introduction}

Inadequate removal of a large number of potentially toxic organic metabolites from the vascular bed into the urine in the course of acute kidney injury (AKI) and chronic kidney disease (CKD) is associated with various clinical symptoms which are often difficult to interpret. Uremic retention solutes are referred to as uremic toxins when they interact with normal biological functions. It is of considerable importance to identify which of the uremic retention solutes are actually uremic toxins and what pathomechanisms are involved in their damaging effect on the kidneys and other organs. This would allow better documented confirmation of the suspected association between the clinical symptoms and uremic retention solute/toxin concentrations in biological materials, possible discovery of any missing pathophysiological links between progressive renal failure and loss of function in organs other than the kidneys, and identification of diagnosis- and organ-specific biomarkers for use in clinical practice. In some special patient populations, such as children, pregnant women, those receiving long-term treatment, those with liver or cardiovascular disease, or after organ transplantation, the control of the type and concentrations of organic metabolites responsible for ad-

\section{KARGER}

E-Mail karger@karger.com www.karger.com/nec
Dr. hab. Barbara Lisowska-Myjak

Department of Biochemistry and Clinical Chemistry, Medical University of Warsaw ul. Banacha 1

PL-02-097 Warsaw (Poland)

E-Mail basia.myjak@interia.pl 
ditional clinical symptoms unrelated to the underlying disease presents a major therapeutic challenge [1-9].

The term uremic syndrome refers to the clinical picture produced by accumulation in the body of noxious substances which in health are eliminated into the urine. Although the clinical symptoms of uremic syndrome have been known for decades, our knowledge of the origin, chemical structure and composition of the retained endogenous substances responsible for these symptoms is far from complete. In renal failure, two likely sources of uremic toxins which may influence the severity of clinical symptoms have been suggested. One is the reduced glomerular filtration or renal metabolism and the other damage to nonrenal organs, and the products comprise a variety of compounds specifically related to the metabolic processes and function of different cell types and organs $[1,3,5,8]$.

State-of-the-art investigation techniques developed in recent years have been used in studies aimed at the identification, characterization, analytical determination and evaluation of the biological activity of uremic retention solutes. Now, numerous problems relating to the evaluation of single substances and their panels and their potential role in the diagnosis of organ dysfunctions are being investigated. The main expected outcome of these studies is finding effective treatments to prevent the toxic effects of uremic retention solutes at different stages of AKI and CKD $[2,10,11]$.

\section{Classification of Uremic Compounds}

Based on 85 studies published in the years 1968-2002 and evaluating over 500,000 patients, the European Uremic Toxin (EUTox) Work Group listed substances with presumed or proven biological activity, the accumulation of which in the body resulted from end-stage renal failure. Newly identified substances are added to the list on an ongoing basis providing an increasingly complex picture of their potential toxicity $[6,8,11-13]$.

Uremic retention solutes present a great variety of properties which makes their accurate classification extremely difficult. They make up a group with numerous members that differ in their water solubility, proteinbinding capacity, molecular weight, pattern of removal by dialysis, biological properties and potential to produce clinical symptoms. In published studies, molar concentrations of uremic retention solutes ranged from a few picomoles per liter (for interleukins) to micromoles per liter (for phenylacetic acid). The highest mass concentra- tion was detected for acute-phase macromolecule $\alpha 1$-acid glycoprotein $[3,5,12-14]$. The most common classification of uremic compounds into 3 groups proposed by EUTox is by molecular weight, protein-binding capacity and removal pattern by dialysis $[3,5,12,13,15]$.

\section{Low-Molecular-Weight Water-Soluble Uremic Toxins}

This group consists of small molecules (molecular weight $<500 \mathrm{Da}$ ) that are soluble in water and easily removed by any dialysis strategy. Low-molecular-weight organic compounds may occur in free water-soluble form or bound to plasma proteins, which alters the function of both the toxin and the transporter protein. Of the $90 \mathrm{~mol}-$ ecules evaluated by EUTox, 68 were found to be members of this group, the most common compounds being ADMA (asymmetric dimethylarginine), creatine, creatinine, hyaluronic acid, guanidine, guanidinoacetate, guanidinosuccinate, oxalate, SDMA (symmetric dimethylarginine), urea and uric acid.

\section{Protein-Bound Solutes}

Twenty-five uremic retention solutes, i.e. $27.8 \%$ of all uremic compounds evaluated by EUTox, are included in the protein-bound solutes group. Although the molecular weight of most members of this group is less than $500 \mathrm{Da}$, because of their protein-binding capacity they are recognized as 'difficult to remove' by dialysis. The main protein-bound solutes include advanced glycation end products (AGEs), carboxy methyl propyl furanpropionic acid, cytokines, interleukins, tumor necrosis factor- $\alpha$ (TNF- $\alpha$ ), dimethylguanidines, hippuric acid, homocysteine, indole-3-acetic acid, indoxyl glucuronide, indoxyl sulfate (IS), kynurenic acid, kynurenine, leptin, phenolic compounds, $p$-cresyl sulfate ( $p$-CS), $p$-cresyl glucuronide, phenol sulfate, phenol glucuronide, phenylacetic acid, quinolinic acid and retinol-binding protein.

\section{Middle-Molecular-Weight Molecules}

The molecular mass of middle-molecular-weight molecules is above $500 \mathrm{Da}$. So far more than 50 such compounds have been found to have a cause-and-effect relationship with the origin and development of many pathophysiological processes. Members of this group include adiponectin, cystatin C, leptin, motilin, al-acid glycoprotein, a1-microglobulin, endothelin, ghrelin, osteocalcin, atrial natriuretic peptide, prolactin, retinol-binding protein, $\beta 2$-microglobulin, cholecystokinin and vasoactive intestinal peptide.

The EUTox classification does not describe the toxicity of the compounds listed and so far there has been no 
effective method of such characterization. It has been suggested that further more precise classification of the compounds should take into consideration any similarities between their chemical structures, common biological or organ function, and the anatomical site of their origin.

\section{Classification of Uremic Compounds Based on Similarities in Chemical Structure}

Guanidine compounds are urea derivatives and include $\alpha$-keto- $\delta$-guanidinovalericacid, $\alpha-\mathrm{N}$-acetylarginine, ADMA, argininic acid, $\beta$-guanidinopropionic acid, creatine, creatinine, $\gamma$-guanidinobutyric acid, guanidine, guanidinoacetic acid, guanidinosuccinic acid, methylguanidine, SDMA and taurocyamine. Although the guanidines are members of one group of small water-soluble compounds with physicochemical characteristics resembling those of urea and creatinine, many guanidine compounds have been shown to have a larger distribution volume compared to urea, which may reduce their effective removal from the body. Further uremic compound groups based on similarities in chemical structure $[2,5$, 15] are listed below:

- Purine derivatives: cytidine, hypoxanthine, xanthine and uric acid

- Pyrimidine derivatives: thymine, orotic acid, orotidine and uridine

- Methyl amine derivatives: methylamine, dimethylamine and trimethylamine

- Phenyl derivatives: 2-methoxyresorcinol, phenol, hydroquinone and $p$-cresol

- Indole derivatives: kinurenine, indole-3-acetate, kynurenic acid, melatonin, IS and quinolinic acid

\section{AGEs as an Example of a Classification of Uremic Compounds by Their Metabolic Source}

AGEs are the result of the chemical chain reaction following the initial glycation. Increases in their concentrations have been linked with the induction of biochemical processes (oxidative stress) and the development of many disorders (mostly chronic inflammatory diseases). AGEs may be formed outside the body or internally through normal metabolism and aging. They are absorbed by the body during digestion with about 30\% efficiency. Large AGE proteins are unable to enter the Bowman capsule. Normal cell proteolysis of AGEs produces AGE peptides

Uremic Toxins and Their Effects on

Multiple Organ Systems and 'AGE-free adducts' (AGE adducts bound to single amino acids) which, after being released into the plasma, can be excreted in the urine. The breakdown products of AGE are more aggressive than the AGE-proteins from which they are derived. According to EUTox the following members of this group have been classified as potential toxins: 3-deoxyglucosone, fructoselysine, glyoxal, methylglyoxal, Ne-carboxymethyllysine (CML), Necarboxyethyllysine (CEL) and pentosidine. Inadequate elimination of AGEs at different stages of renal failure may lead to their accumulation in the body. Normal plasma concentrations of glycation-free adducts are increased 5 -fold in patients with mild chronic renal failure who do not require dialysis, 18 -fold in patients on peritoneal dialysis and 40-fold in patients treated with hemodialysis. In CKD patients, CML and CEL are the two main glycation-free adducts. Experimental studies evaluating AGEs have shown their low toxicity at uremic concentrations, but considering their proven part in many physiological processes, these are the panels of AGEs accumulated in the body in the course of renal impairment that should be systematically analyzed, rather than selected individual members of this group $[5,9]$.

\section{Uremic Compounds Produced in the Intestine as an Example of Their Classification by Organ of Origin}

The isolated intestinal space facilitates the identification, characterization and development of treatments to inhibit the production and absorption of toxic substances reaching the intestine with ingested food and produced within the intestine by bacteria. The role of the large intestine in the production of toxic uremic compounds was highlighted by studies comparing the qualitative and quantitative composition of toxic uremic compounds in the plasma of hemodialyzed patients with the large intestine intact and patients in whom the large intestine had been removed. Using this study design, some uremic compounds were found exclusively in the patients with the intact large intestine, including uremic compounds produced by bacteria. Chemical identification of many compounds produced in the large intestine is now considerably limited by the absence of relevant benchmarks available for comparison in the databases. So far two groups of chemical compounds produced by colon microbes have been identified: indole compounds (IS, indoxyl glucuronide, 5-hydroxyindole, indole-3-propionic acid) and phenyl compounds ( $p$-CS, $p$-cresol glucuronide, phenyl sulfate, phenyl glucuronide, $\alpha-\mathrm{N}$ - 
phenylacetyl-L-glutamine, phenylpropionylglycine, cinnamoylglycine, 4-ethylphenyl sulfate, hippuric acid). The biological activity of colon-related uremic compounds has been linked with adverse effects on the function of the kidneys and other organs (liver, brain, endothelium) $[10,16-18]$.

\section{Uremic Compounds Related to Endothelium}

The activity of uremic compounds (listed below in parentheses) has been associated with the following common mechanisms of endothelial dysfunction $[5,7,14]$ :

(1) Increased manifestations of atherosclerosis, including leukocyte activation (guanidine derivatives, AGEs, $p$-CS, platelet diadenosine polyphosphates, IS), endothelial adhesion (IS, vascular cell adhesion molecule-1), migration and proliferation of vascular smoothmuscle cells (IS, AGEs), thrombosis and plaque destabilization (von Willebrand factor, thrombomodulin, plasminogen activatorinhibitor 1 , matrix metalloproteinases), and a decrease in nitric oxide availability (ADMA, AGEs, circulating endothelial microparticles).

(2) Increasing rigidity of blood vessels resulting from the loss of vessel wall compliance (ADMA, AGEs).

(3) Vascular calcification (inorganic phosphate, reactive oxygen species, tumor necrosis factor, leptin).

(4) Abnormalities of vascular repair (IS, some guanidine compounds).

\section{Uremic Compounds Involved in the Acute-Phase Processes}

The group of uremic compounds involved in the acute-phase processes includes those which can be associated with a generalized increase in the inflammatory response in patients with decreased renal function. Impaired renal function may enhance the overall inflammatory response because of the decreased renal clearance of compounds with antioxidant, anti-inflammatory and vasodilating properties, which in health can be filtered by the glomeruli (molecular weight $<58 \mathrm{kDa}$ ), such as proinflammatory cytokines, $\alpha_{1}$-acid glycoprotein, neopterin and calcitonin. There is increasing evidence confirming the role of impaired intestinal barrier function and potential toxins either produced in the intestine (phenoles, indoles) or introduced with the ingested food via the intestine (AGEs). Declining renal function may also affect the level of several acute-phase proteins with large molecules
(CRP, $\alpha_{2}$-macroglobulin, fibrinogen, myeloperoxidase) which are directly or indirectly involved in inflammation.

In patients with $\mathrm{CKD}$, inflammation may be closely related to accelerated atherogenesis, protein-energy malnutrition and anemia via different mechanisms. Inflammation plays a key role in mediating CKD progression in response to infectious and noninfectious kidney damage. Increased levels of inflammatory markers predict poor outcome in patients with ESRD and chronic renal failure $[10,16,19-22]$.

\section{Strategy to Determine the Toxicity of Retained Compounds}

So far only a few organic metabolites have been found with the properties which would allow their classification as uremic toxins. Still, other retained compounds may be potentially toxic and hence useful as laboratory markers for evaluation of impaired glomerular filtration. With the abundance of new information about the potential toxicity of different uremic compounds, it becomes increasingly important to determine the principles guiding the comparison of data from different research centers.

Urea and creatinine classified by EUTox as belonging to the same group of low-molecular-weight water-soluble uremic toxins are the most common biomarkers for assessment of renal failure. Historically, the term 'uremia' denoted increased serum concentrations of urea, which, because of its abundance, has been routinely used to assess the effectiveness of dialysis. On the other hand, in current clinical practice, of all the retained uremic solutes measured creatinine is the only one to evaluate the biochemical/biological and hence toxic effects of renal dysfunction. Paradoxically, unlike in the case of other potentially toxic compounds, there have been only a few published studies confirming the ability of either urea or creatinine to induce adverse biochemical and physiological effects. There have been questions concerning the diagnostic value of the eGFR equations (estimated glomerular filtration rate) based on serum creatinine measurements and used to diagnose the stages and progression of renal dysfunction. The evidence so far collected confirms that the eGFR values show a very weak and diverse correlation with other proven uremic toxins. Hence, the current criteria based exclusively on eGFR seem inadequate to justify the decision to start dialysis $[2-4,11,12,15,16]$.

In the last decade numerous previously unknown uremic compounds have been identified and their associa- 
tions with specific pathophysiological mechanisms have been established. However, their potential toxicity remains to be elucidated with meticulous observation of clearly formulated rules guiding the process $[1-3,5,6,8$, $12,15,17,18,23-25]$.

(1) In vitro studies of the biological effects of candidate compounds are a basis for their further identification in epidemiological and clinical studies. The need for the use of specific cells for the purposes of disease modelling has been underlined, such as leukocytes to study compromised immune defense or oxidative stress, endothelial cells for cardiovascular disease, smooth-muscle cells for progression of atherosclerosis, hepatocytes for disturbed metabolism, fibroblasts for fibrosis or osteoblasts for renal osteodystrophy. When possible, human cells should be used, and the animal cell models should be restricted to the species for which the relevance to conditions in humans has already been proven.

(2) Appropriate selection of analytical methods is a prerequisite for accurate comparison of the concentrations of uremic toxins measured in vitro and in vivo in biological material. The method chosen depends of the sensitivity of detection of particular compounds. A number of isolation and detection techniques have been used for the quantification of retained uremic compounds, including chromatographic methods (ion exchange chromatography, gas chromatography, HPLC), spectrophotometry, fluorometry, chemiluminescence, nephelometry, radioimmunometry, nuclear magnetic resonance and mass spectrometry). Determination methods should be reproducible and the ranges of quantitative measurements precisely defined and carefully analyzed when applied to different patient populations.

(3) Proteomic and genomic studies are valuable research tools used both to evaluate known uremic compounds and to search for and identify new substances potentially affecting selected conditions and organ functions. These techniques allow differentiation of retained uremic solutes as they occur in particular conditions and patient populations.

(4) Formation of metabolites of potential uremic compounds is a cause of their altered toxicity resulting from altered initial biological activity. An example of this phenomenon is $p$-cresol generated by intestinal bacteria as a metabolite from the amino acids tyrosine and phenylalanine, which is an inhibitor of leukocyte function, while $p$-CS demonstrates proinflammatory effects by activating leukocyte free radical production. Exposure to the uremic milieu in the course of renal failure may also cause irreversible alterations in the structure and function of nu- merous peptides (heterogenous group of AGEs, advanced oxidation protein products, carbamylated proteins).

(5) Differences in protein-binding capacity as well as the proportions of the free fraction and the proteinbound fraction may modify the toxicity of uremic compounds. To date, studies concerning the toxicity of protein-bound toxins have focused on the problems of their removal with commonly used dialysis strategies, which is more difficult and less effective than the elimination of nonbound solutes of similar molecular weight. A comparison of 12 protein-bound uremic toxins showed changes in their protein-binding ratios. IS, $p$-CS and CMPF (3-carboxy-4-methyl-5-propyl-2-furanopropionic acid) could not be removed efficiently by hemodialysis due to their high protein-binding ratios. A question arises as to whether there are any differences in the toxicity between the free and the protein-bound forms of the same compounds and what their role as laboratory parameters is. In CKD patients, the albumin-binding capacity is decreased and associated with the severity of their renal disease and accumulation of uremic albumin-bound retention solutes. Numerous mechanisms which may be responsible for decreased binding to plasma proteins in CKD patients have been taken into consideration, including hypoalbuminemia, accumulation of endogenous substances competing for binding on serum albumin and conformational changes in the albumin molecule.

Albumin-binding capacity may be a valuable laboratory tool to assess the accumulation of free uremic compounds. Albumin is an important transporter protein for water-insoluble drugs and toxins. Over $90 \%$ of uremic compounds in the plasma are albumin-bound but there is equilibrium between the strongly protein-bound and the free forms, which, however, may be rapidly disturbed. The best-described albumin-bound uremic compounds are IS and $p$-CS, which circulate noncovalently bound to albumin and compete for the same binding site on the albumin molecule.

(6) Biological interaction of retained uremic compounds - interactions between retained uremic toxins have been demonstrated in the course of kidney disease $[11,15,18]$ :

- Soluble guanidines are responsible for the production of TNF- $\alpha$ and interleukin-6 (two middle molecules).

- IS induces the synthesis of free radicals in the cells of renal tubular and mesangial cells activating the NF- $\mathrm{kB}$ pathway.

- ADMA increases may result from the inhibition of the enzyme dimethylarginine dimethylaminohydrolase (DDAH) by hyperhomocysteinemia (according to 
EUTox homocysteine is a protein-bound compound). Decreases in blood homocysteine levels may affect the levels of both DDAH and ADMA.

- SDMA and its structural analogue ADMA are members of the same group of water-soluble compounds and have a similar biological effect of inhibiting NO synthesis although their mechanism of action differ.

- In vitro, $p$-CS induces leukocyte free radical production, which was enhanced when $p$-cresyl glucuronide was added but $p$-cresyl glucuronide alone had no effect on leukocyte oxidative burst.

\section{An Ideal Parameter or Panel of Parameters to Assess the Toxicity of Retained Compounds}

In health, the renal glomerular filter cleanses the body of molecules with weights up to $58 \mathrm{kDa}$. All substances retained in the body as a result of renal dysfunction are potential uremic toxins. A retained substance to be classified as uremic toxin must meet the following criteria [2, $4,5,8,11]$ :

(1) The chemical structure and composition should be identifiable and the substance should be quantifiable in biological fluids using a recognized methodology.

(2) Concentrations in the biological fluids or tissues of patients with renal dysfunction should significantly exceed those in nonuremic subjects.

(3) Increases in the concentration in the blood or tissue should correlate with the clinical manifestations.

(4) The association between the biological activity and the clinical manifestations should be demonstrable in in vivo, ex vivo and in vitro test systems.

The following two sections outline areas in need of elucidation through further studies.

\section{Which Uremic Toxins Have a Similar}

\section{Pathophysiological Role in Organ Damage, and at}

\section{What Concentration Ranges?}

An example here is the involvement of many uremic toxins in the development of cardiovascular disease. Although IS and $p$-CS are included by the EUTox in the same group of uremic compounds and the source of their synthesis, i.e. intestinal bacteria, is the same, they differ in the mechanism and extent of their effect on the development of cardiovascular disease [8]. A question arises as to whether the quantification of these compounds provides identical diagnostic information, or whether they are complementary sources of information about pathologi- cal effects produced via different biological pathways. To answer this question it is necessary to classify the retained uremic compounds by their pathophysiological role rather than by chemical structure alone. A need emerges for determining not only the concentration limits associated with the toxicity of the individual compounds, but also to quantify the limit for the sum of the concentrations of all substances involved in the same pathological process. The search for new uremic compounds and combining them into panels of substances involved in the same pathophysiological processes seems to offer a novel approach to identifying and explaining so far unknown unexplored specific effects of endogenous compounds on the body organs and systems.

\section{Is It Possible to Assess Dysfunction of a Body Organ}

Based on the Measurements of One Compound or

Should a Panel of Compounds Be Used?

Toxicity assessment is a complex process because not just one but several retained compounds may be simultaneously involved in the same biological and metabolic processes. Nearly all body organs and systems are affected by the toxicity of uremic compounds retained in the course of renal failure, including the cardiovascular system (atheromatosis, arteriosclerosis, decreased diastolic compliance, hyper/hypotension, pericarditis), central nervous system (concentration disorder, cramps, dementia, depression, fatigue, headache, motor weakness, polyneuritis, reduced sociability, restless legs, sleep disorders, stupor), hematological and coagulation disorders (anemia, bleeding disorders, overcoagulation), immune system (inadequate antibody formation, inflammation stimulation, susceptibility to cancer, susceptibility to infections), endocrine system (dyslipidemia, glucose intolerance, stunned growth, hyperparathyroidism, hypogonadism, erectile dysfunction, decreased libido), bone disease (adynamic bone disease, impaired calcitriol metabolism, osteitis fibrosa, osteomalacia, osteoporosis), skin (melanosis, pruritus), gastro-intestinal system (anorexia, dyspepsia, gastrointestinal ulceration, hiccup, nausea, vomiting, pancreatitis) and respiratory system (pleuritis, emphysema, sleep apnea syndrome).

Classification of uremic compounds involved in the processes damaging vascular endothelium demonstrates that they maybe small water-soluble compounds (ADMA, guanidinoacetic acid, methylguanidine), protein-bound molecules (AGE, dinucleotide polyphosphates, homocysteine, IS sulfate, $p$-CS, phenylacetic acid) and middle molecules (leptin, TNF- $\alpha$ ). Identification of reliable biomarkers or their panels needs careful consideration of 
Table 1. Links between retained uremic compounds and early kidney damage and impaired function of other organs

\begin{tabular}{|c|c|c|c|}
\hline Kidney & $\begin{array}{l}\text { AKI } \\
\text { CKD }\end{array}$ & $\begin{array}{l}\text { Sudden renal tubular and endothelial injury } \\
\text { Progression of renal failure }\end{array}$ & $\begin{array}{l}19,23,26 \\
19,20,27\end{array}$ \\
\hline $\begin{array}{l}\text { Kidney-heart } \\
\text { axis }\end{array}$ & $\begin{array}{l}\text { Cardiorenal syndrome - } 5 \text { types of different } \\
\text { interactions between chronic dysfunctions of the } \\
\text { heart or kidneys which can induce acute or } \\
\text { chronic dysfunction of other organs }\end{array}$ & $\begin{array}{l}\text { Possible link between kidney function and } \\
\text { cardiovascular risk }\end{array}$ & $\begin{array}{l}2,3,5,9,13,14 \\
28,29\end{array}$ \\
\hline $\begin{array}{l}\text { Kidney- } \\
\text { intestinal } \\
\text { mucosal } \\
\text { barrier axis }\end{array}$ & $\begin{array}{l}\text { Impaired function of key proteins of the } \\
\text { intestinal epithelial tight junction in uremia }\end{array}$ & $\begin{array}{l}\text { Translated into circulation bacteria and their } \\
\text { products affect the activation of the innate } \\
\text { immune system }\end{array}$ & $\begin{array}{l}16,17,24,30 \\
31,33\end{array}$ \\
\hline $\begin{array}{l}\text { Kidney-lung } \\
\text { axis }\end{array}$ & Increased lung vascular permeability in AKI & Mediators of AKI-induced lung changes & 37 \\
\hline
\end{tabular}

their concentrations in biological materials, biological activity and usefulness for effective diagnosis of consecutive stages of endothelial dysfunction $[4,5]$.

\section{Retained Uremic Compounds as Markers of Organ Damage}

Table 1 presents the current literature data concerning the role of accumulated uremic toxins in the onset and development of acute and chronic renal dysfunction, and the relationship between kidney disease and the dysfunction of other organs. It has been suggested that uremic toxins promote progression of renal failure by damaging tubular cells and their overload accelerates the loss of kidney function, glomerular sclerosis and tubulointerstitial injury $[26,27]$.

Better understanding of the role of endogenous uremic toxins as a likely factor involved in the so far unclear pathomechanism of the relationship between renal disease and cardiovascular disease is needed for developing effective treatment strategies $[2,3,5,9,13,14$, 28, 29].

Pathogen overgrowth (dysbiosis) with additional elimination of creatinine through the intestinal wall is common in the course of CKD. Impaired function of the intestinal mucosal barrier may explain the persistence of systemic inflammation in the course of CKD $[16,17,21$, 30-33].

The liver and kidneys together comprise an organ system responsible for the removal of toxic compounds from the body. Renal function loss in patients with cirrhosis has been associated with a worse prognosis $[34,35]$.

Accumulation of uremic toxins may cause cerebral endothelial dysfunction and contribute to cognitive disorders in CKD [36]. Uremic toxins in AKI may modulate lung dysfunction, susceptibility to lung injury, or both [38].

\section{Treatments to Prevent Damage from Uremic Toxins}

According to recent reports $[10,15,18]$, effective removal of uremic compounds improves treatment outcomes and survival in patients with renal failure. Two main directions of research for improved treatment methods have emerged:

(1) More effective removal of uremic toxins by dialysis as the protein-bound toxins cannot be eliminated using the current dialysis strategies.

(2) Use of pharmacological agents to interfere with the production and absorption of colon-derived solutes. 
The suggested approaches are outlined below $[7,10$, $11,15,18,38]$ :

- Influencing the growth and metabolism of intestinal bacteria by the use of probiotics (products containing bifidobacteria), prebiotics (resistant starch, oligofructose-enriched inulin) and antibiotics.

- Diet modification. Colon-derived uremic solutes such as IS and $p$-CS generated by intestinal bacteria are derived from dietary amino acids (phenylalanine, tryptophan). Additionally, intestinal protein absorption is disturbed in renal failure with the resulting increase in the number of intestinal substrates for colon microbes. Reducing dietary protein intake and increasing the amount of dietary fiber may be an easy way to decrease the production of colon-derived uremic solutes.

- Preventing constipation, including the use of laxatives, as decreasing the duration of protein transit in the large intestine reduces the metabolism of amino acids to potentially toxic uremic compounds.

- Sorbent therapy. Intestinal adsorption of uremic compound precursors inhibits their further conversion into active uremic toxins. Decreasing serum IS levels using the intestinal carbon-based sorbent AST-120 (Kremezin) produces reduction of oxidative stress in the kidney, improved renal function and less histological damage in vivo.

\section{Conclusion}

A new critical review of the diagnostic usefulness of such recognized biomarkers for kidney failure as creatinine and urea which have been used for decades indicates that, contrary to the initial assumptions, these measurements do not fully reflect the combined toxicity due to the presence of other endogenous organic me- tabolites. Considering the increasing numbers of patients with diagnosed CKD and AKI, there is an urgent need to search for new biological markers of greater diagnostic specificity to guide treatments to prevent or at least slow down organ damage in the course of kidney failure. Numerous studies have been published recently which prove the toxicity of uremic compounds but are still unable to elucidate the exact mechanism of their relationship with clinical symptoms of organ malfunction. They highlight numerous problems related to the chemical identification of these compounds and the assessment of their biological activity, but at the same time pose a number of questions which, when answered, would enhance our present knowledge of the physiology and pathology of metabolic processes. The present review demonstrates that so far only a fraction of these questions has been answered. Each of the markers presents unique biological and kinetic properties and their uremic toxicity cannot be definitively described with the currently available data. Also, a new classification of uremic compounds, by mechanism of action, is needed. The most valuable extensive studies initiated in the field of uremic toxin research by the EUTox Work Group should be continued in many research centers and the findings archived according to the strictly defined rules. Modern technologies have allowed the formulation of an initial strategy for the identification and chemical characterization of uremic toxins. It is to be hoped that further research will provide new knowledge of value for the clinical practice.

\section{Acknowledgements}

This work was supported by a grant (DEC-2011/01/B/ NZ7/00648) from the National Science Centre (Poland).

\section{References}

1 Herget-Rosenthal S, Glorieux G, Jankowski J, Jankowski V: Uremic toxins in acute kidney injury. Semin Dial 2009;22:445-448.

- Vanholder R, Meert N, Schepers E, Glorieux G: Uremic toxins: do we know enough to explain uremia? Blood Purif 2008;26:77-81.

-3 Vanholder R, Glorieux G, De Smet R, Lameire N, European Uremic Toxin Work Group (EUTox): New insights in uremic toxins. Kidney Int 2003;63:S6-S10.

44 Glorieux G, Schepers E, Vanholder RC: Uremic toxins in chronic renal failure. Prilozi 2007;28:173-204.
5 Vanholder R, Baurmeister U, Brunet P, Cohen G, Glorieux G, Jankowski J, European Uremic Toxin Work Group (EUTox): A bench to bedside view of uremic toxins. J Am Soc Nephrol 2008;19:863-870.

6 Vanholder R, Laecke SV, Glorieux G: What is new in uremic toxicity? Pediatr Nephrol 2008; 23:1211-1221.

7 Eloot S, Schepers E, Barreto DV, Barreto FC, Liabeuf S, van Biesen S, Verbeke F, Glorieux G, Choukroun G, Massy Z, Vanholder R: Estimated glomerular filtration rate is a poor predictor of concentration for a broad range of uremic toxins. Clin J Am Soc Nephrol 2011; 6:1266-1273.

8 Liabeuf S, Drueke TB, Massy ZA: Proteinbound uremic toxins: new insight from clinical studies. Toxins 2011:3:911-919.

-9 Zhu J, Yang K, Jing Y, Du R, Zhu Z, Lu L, Hang R: The effects of low-dose nepsilon -(carboxymethyl)lysine (CML) and nepsilon(carboxyethyl)ysine (CEL), two main glycation free adducts considered as potential uremic toxins, on endothelial progenitor cell function. Cardiovasc Diabetol 2012;11:9099. 
10 Meyer TW, Hosetter TH: Uremic solutes from colon microbes. Kidney Int 2012;81: 949-954.

11 Meijers BKI, Evenepoel P: The gut-kidney axis: indoxyl sulfate, $p$-cresyl sulfate and CKD progression. Nephrol Dial Transplant 2011; 26:759-761.

-12 Yavuz A, Tetta C, Ersoy FF, D'intini V, Ratanarat R, De Cal M, Bonello M, Bordoni V, Salvatori G, Andrikos E, Yakupoglu G, Levin NW, Ronco C: Uremic toxins: a new focus on old subject. Semin Dial 2005;18:303211.

13 Duranton F, Cohen G, De Smet R, Rodriguez M, Jankowski J, Vanholder R, Argiles A, European Uremic Toxin Work Group: Normal and pathologic concentrations of uremic toxins. J Am Soc Nephrol 2012;23:1258-1270.

-14 Brunet P, Gondouin B, Duval-Sabatier A, Dou L, Cerini C, Dignat-George F, JourdeChiche N, Argiles A, Burtey S: Does uremia cause vascular dysfunction? Kidney Blood Press Res 2011;34:284-290.

-15 Neirynck N, Vanholder R, Schepers E, Eloot S, Pletinck A, Glorieux G: An update on uremic toxins. Int Urol Nephrol 2013;45:139-150.

-16 Aronov PA, Luo FJG, Plummer NS, Quan Z, Holmes S, Hostetter TH, Meyer TW: Colonic contribution to uremic solutes. J Am Soc Nephrol 2011;22:1769-1776.

17 Hauser AB, Stinghen AEM, Gonçalves SM, Bucharles S, Pecoits-Filho R: A gut feeling on endotoxemia: causes and consequences in chronic kidney disease. Nephron Clin Pract 2011;118:c165-c172.

18 Neirynck N, Glorieux G, Schepers E, Pletinck A, Dhondt A, Vanholder R: Review of protein-bound toxins, possibility for blood purification therapy. Blood Purif 2013;35(suppl 1):45-50.
19 Panichi V, Migliori M, De Pietro S, Taccola D, Bianchi AM, Norpoth M, Metelli MR, Giovannini L, Tetta C, Palla R: C reactive protein in patients with chronic renal diseases. Ren Fail 2001;23:551-562.

20 Tesch GH: Review: serum and urine biomarkers of kidney disease: a pathophysiological perspective. Nephrology 2010;15:609-616.

21 Sun CY, Hsu HH, Wu MS: p-Cresol sulfate and indoxyl sulfate induce similar cellular inflammatory gene expression in cultured proximal renal tubular cells. Nephrol Dial Transplant 2013;28:70-78.

22 Vaziri ND, Yuan J, Rahimi A, Ni Z, Said H, Subramanian VS: Disintegration of colonic epithelial tight junction in uremia: a likely cause of CKD-associated inflammation. Nephrol Dial Transplant 2012;27:2686-2693.

23 Cohen G, Raupachova J, Hörl WH: The uraemic toxin phenylacetic acid contributes to inflammation by priming polymorphonuclear leucocytes. Nephrol Dial Transplant 2013;28: 421-429.

24 Klammt S, Wojak HJ, Mitzner A, Koball S, Rychly J, Reisinger EC, Mitzner S: Albuminbinding capacity $(\mathrm{ABiC})$ is reduced in patients with chronic kidney disease along with accumulation of protein-bound uremic toxins. Nephrol Dial Transplant 2012;27:23772383.

5 Itoh Y, Ezawa A, Kikuchi K, Tsuruta Y, Niwa T: Protein-bound uremic toxins in hemodialysis patients measured by liquid chromatography/tandem mass spectrometry and their effects on endothelial ROS production. Anal Bioanal Chem 2012;403:1841-1850.

26 Lisowska-Myjak B: Serum and urinary laboratory markers in acute kidney injury. Blood Purif 2010;29:357-365.

-27 Satoh M, Hayashi H, Watanabe M, Ueda K, Yamato H, Yoshioka T, Motojima M: Uremic toxins overload accelerates renal damage in a rat model of chronic renal failure. Nephron Exp Nephrol 2003;95:e111-e118.

28 Goh CY, Vizzi G, De Cal M, Ronco C: Cardiorenal syndrome : complex series of combined heart/kidney disorders. Contrib Nephrol 2011;174:33-45.
29 Chan EJ, Dellsperger KC: Cardiorenal syndrome: the clinical cardiologists' perspective. Cardiorenal Med 2011;1:13-22.

30 Peng YS, Lin YT, Hung KY, Wang SM: Effects of indoxyl sulfate on adherens junctions of endothelial cells and the underlying signaling mechanisms. J Cell Biochem 2012;113:10341043.

31 Anders HJ, Andersen K, Stecher B: The intestinal microbiota, a leaky gut, and abnormal immunity in kidney disease. Kidney Int 2013; 83:1010-1016.

32 Johnson D: Evaluation of renal function: use of serum creatinine concentration to assess level of kidney function. Nephrology 2005; 10:S133-S176.

33 Vaziri ND: CKD impairs barrier function and alters microbial flora of the intestine: a major link to inflammation and uremic toxicity. Curr Opin Nephrol Hypertens 2012;21:587592

- 34 Wadei HM, Mai ML, Ahsan N, Gonwa TA Hepatorenal syndrome: pathophysiology and management. Clin J Am Soc Nephrol 2006; 1: 1066-1079.

35 Lhotta K: Beyond hepatorenal syndrome: glomerulonephritis in patients with liver disease. Semin Nephrol 2002;22:302-308.

-36 Bugnicourt JM, Godefroy O, Chillon JC Choukroun G, Massy ZA: Cognitive disorders and dementia in CKD: the neglected kidney-brain axis. J Am Soc Nephrol 2013:24: 353-363.

37 Rabb H, Wang Z, Nemoto T, Hotchkiss J, Yokota N, Soleimani M: Acute renal failure leads to dysregulation of lung salt and water channels. Kidney Int 2003;63:600-606.

38 Vitetta L, Gobe G: Uremia and chronic kidney disease: the role of the gut microflora and therapies with pro-and prebiotics. Mol Nutr Food Res 2013;57:824-832. 